\title{
EFEITO DO FÓSFORO, FUMIGAÇÃO DO SUBSTRATO E FUNGO MICORRÍZICO ARBUSCULAR SOBRE O CRESCIMENTO DE PLANTAS DE MAMOEIRO(1)
}

\author{
M.T. A. MINHONI(2) \& P. A. M. AULER ${ }^{(3)}$
}

\begin{abstract}
RESUMO
Estudou-se o efei to da inoculação com o fungo micorrízico arbuscular (FMA), Glomus macrocarpum, da fumigação do substrato e da adição de fósforo solúvel $\left(60,120,240\right.$ e $480 \mathrm{mg} \mathrm{kg}^{-1}$ de $P$ no solo) sobre as variáveis altura, número de fol has e diâmetro do caule de plantas de mamoeiro cv. Sunrise Solo. O FMA e doses crescentes de fósforo, i soladamente, exerceram efeitos si gnificativos sobre essas variáveis. Não houve efeito si gnificativo do fator fumigação do substrato. 0 efeito da inoculação foi mais acentuado no tratamento com adição de $60 \mathrm{mg} \mathrm{kg}^{1}$ de P no solo. A inoculação com G. macrocarpum reduziu a necessidade de fósforo para o mamoeiro, tanto que as variáveis estudadas em plantas inoculadas na ausência de adubação fosfática não diferiram de plantas não inoculadas em

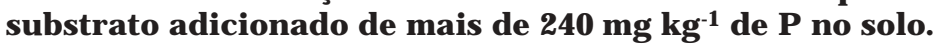

Termos de indexação: Carica papaya, Glomus macrocarpum, altura, diâmetro de caule, número de folhas, doses de fósforo, desinfecção do substrato.

\section{SUMMARY: EFFECT OF PHOSPHORUS, SOIL FUMIGATION AND MYCORRHIZAL COLONIZATION ON PAPAYA GROWTH}

The effects of inoculation with arbuscular mycorrhizal fungus (AMF), substratum fumigation, and addition of soluble phosphate on papaya plant cultivar "Sunrise Solo" parameters (height, leaf number, and stem diameter) were studied. Separate treatments with AMF inoculation (Glomus macrocarpum) and increasing phosphorus levels (60, 120,

\footnotetext{
(1) Trabalho apresentado no XXI Congresso Brasileiro de Microbiologia, Foz do I guaçu (PR), 21 a 25 de outubro de 2001. Recebido para publicação em agosto de 2001 e aprovado em junho de 2003.

(2) Professora do Departamento de Produção Vegetal, Faculdade de Ciências Agronômicas, Universidade Estadual Paulista - UNESP. Caixa Postal 237, CEP 18603-970 Botucatu (SP). E-mail: marliminhoni@fca.unesp.br

(3) Pesquisador do Instituto Agronômico do Paraná - IAPAR. Estação Experimental de Paranavaí, Caixa Postal 564, CEP $87701-970$ Paranavaí (PR). E-mail: aulerp@iapar.br
} 
240, and $480 \mathrm{mg} \mathrm{kg}^{-1}$ of $\mathrm{P}$ in soi I) influenced theseparameters si gnificantl y, whilethefactor substratum fumigation had no si gnificant effect. The inoculati on effect was most expressi ve when $60 \mathrm{mg} \mathrm{kg}^{-1}$ of $\mathrm{P}$ in soil was added. Such was thereduction in phosphor us requirement of papaya by Glomus macrocarpum inoculation, that the studied parameters between inoculated plants without phosphate addition and not-inoculated ones in substratum enriched with over $240 \mathrm{mg} \mathrm{kg}^{-1}$ of $\mathrm{P}$ in soil did not differ.

Index terms: Carica papaya, Glomus macrocarpum, height, stem diameter, leaf number, phosphorus levels, substratum disinfection.

\section{NTRODUÇÃO}

A formação de mudas de frutíferas em viveiros depende, na maioria das vezes, de um tratamento fitossanitário prévio do substrato, para diminuição ou eliminação de sementes de plantas daninhas, pragas e, ou, patógenos. Contudo, esta prática acarreta também a redução ou eliminação de microrganismos benéficos presentes no substrato, tais como os fungos micorrízicos arbusculares (FMAs). Como conseqüência, a introdução de FMA tem efeitos positivos no crescimento de plantas de frutíferas (Kleinschmidt \& Gerdemann, 1972; Menge et al., 1980; Cardoso et al., 1986; Lin, 1986; Soares $\&$ Martins, 2000; Melloni et al., 2000). Em mamoeiro, observaram-se respostas positivas com a inoculação de Glomus macrocarpum (Ramirez et al., 1975; Silva \& Siqueira, 1991), G. mosseae e G. fasciculatum (Mohandas, 1992), G. darum, Gigaspora margarita e Acaul ospora scrobiculata (Trindade et al., 2000), entre outros.

A importância das associações micorrízicas no crescimento, desenvol vimento e nutrição das plantas tem sido enfatizada em várias revisões (Maronek et al., 1981; Lopes et al., 1983; Moreira \& Siqueira, 2002). Nesta associação simbiótica mutualista, muitos fatores estão envolvidos e, dentre estes, o teor de fósforo no substrato de cultivo é fator condicionador para o estabelecimento e funcionalidade do processo.

O objetivo do presente trabalho foi verificar os efeitos da adubação fosfática em várias doses, da fumigação do substrato e da inoculação com o FMA G. macrocarpum sobre a altura, número de fol has e diâmetro do caule de plantas de mamoeiro.

\section{MATERIAL E MÉTODOS}

O experimento foi realizado em condições de casa de vegetação, no Departamento de Produção Vegetal da F acul dade de Ciências Agronômi cas, UNESP, em Botucatu, São Paulo, Brasil. A amostra de solo utilizada na composição do substrato foi de um Latossolo Vermelho álico e epidistrófico textura média (EMBRAPA, 1999), do município de Botucatu (SP). A amostra, col etada a 0-20 cm e peneirada a $2 \mathrm{~mm}$ (peneira ABNT $\mathrm{n}^{\circ} 10$ ), apresentou-se com as seguintes características químicas: $\mathrm{pH}$ em $\mathrm{CaCl}_{2}=$ 4,0; matéria orgânica $=28 \mathrm{~g} \mathrm{dm}^{-3} ; \mathrm{P}=3 \mathrm{mg} \mathrm{dm}^{-3}$; $\mathrm{H}+\mathrm{Al}=6,4 \mathrm{cmol}_{\mathrm{C}} \mathrm{kg}^{-1} ; \mathrm{K}=0,05 \mathrm{cmol}_{\mathrm{C}} \mathrm{kg}^{-1} ; \mathrm{Ca} \stackrel{ }{=}$ $0,20 \mathrm{cmol}_{\mathrm{C}} \mathrm{kg}^{-1} ; \mathrm{Mg}=0,1 \mathrm{cmol}_{\mathrm{C}} \mathrm{kg}^{-1} ; \mathrm{T}=6,6 \mathrm{cmol}_{\mathrm{C}} \mathrm{kg}^{-1}$ e V $=5 \%$. Nas determinações do P, K, Ca, e Mg, a extração foi efetuada em resina (Raij \& Quaggio, 1983).

\section{Fase de bandejas}

Sementes extraídas de frutos hermafroditas de mamoeiro cv. Sunrise Sol o foram lavadas em água corrente para remoção do arilo e secas à sombra. Procedeu-se à semeadura em bandejas de isopor, na proporção de uma semente por célula e cada célula com $10 \mathrm{~cm}^{3}$ de substrato. O substrato utilizado foi uma mistura de vermiculita e terra (3:1). Previamente à semeadura, adicionou-se calcário dolomíticoà terra seca ao ar (PRNT $95 \%$, MgO $18 \%$, CaO $28 \%$, V $92 \%$ ), na proporção de $2,8 \mathrm{~g} \mathrm{~kg}^{-1}$ no solo, e incubou-se por 20 dias, com umidade de $70 \%$ da capacidade efetiva de retenção de água, aproximadamente. Após, procedeu-seà mistura da terra com a vermi culita. O substrato, assim formado, foi submetido à tindalização, colocando-o sob vapor fluente de autoclave, durante duas horas diárias, por três dias consecutivos.

Vinte e dois dias após a semeadura, procedeu-se ao transplante das plântulas, com seus respectivos substratos, para bandejas maiores, $100 \mathrm{~cm}^{3}$ por célula, na proporção de uma plântula por célula. O volume foi completado com o mesmo substrato e, na base de cada célula, adicionaram-se, ou não, $3 \mathrm{~cm}^{3}$ de inóculo do FMA G. macrocarpum (substrato + raízes col onizadas +240 esporos). O inócul o do FMA, da micoteca do Laboratório de Microbiologia Agrícola, ESALQ/USP, Piracicaba (SP), foi multiplicado em rizosfera de milho, em substrato areia: terra (9:1) previamente esterilizado sob vapor fluente de autoclave, durante duas horas diárias, por três dias consecutivos.

Para as plântulas-controle, sem adição de FMA, utilizaram-se $3 \mathrm{~cm}^{3}$ de substrato de cultivo de milho isento de FMA. A solução nutritiva de Hoagland e 
Arnon, sem ferro, diluída a 50 \% em água destilada, foi aplicada por meio de regador de crivo fino. As aplicações foram feitas aos dez e dezessete dias do transplante e, a partir daí, a cada dois dias. O manejo da água foi diário, com água destilada, procurando-semanter a umidade do substrato num nível adequado, evitando-se perdas por lixiviação.

Nesta fase de bandeja, o delineamento foi casualizado, com dois tratamentos (com esem F MA) e 16 repetições. Determinaram-se a altura e o número de fol has das plantas de mamoeiro aos 0,7 , 14, 20, 28, 35 e 42 dias da inoculação, ou não, com o FMA.

\section{Fase de vasos}

Aos 49 dias da inoculação, procedeu-se ao transplante para vasos que continham terra fumigada ou não fumigada, na proporção de uma planta por vaso. A fumigação, previamente ao transplante, foi feita com brometo de metila (98\%) mais cloropicrina (2\%). Para tanto, a amostra de solo foi umedecida até $70 \%$ da capacidade efetiva de retenção de água, aproximadamente, e disposta sob lona plástica, por $72 \mathrm{~h}$, para a ação do agente fumigante, dose de $393 \mathrm{~cm}^{3} \mathrm{~m}^{-3}$ de solo. Após esse período, retirou-se a lona plástica e a amostra de solo foi ventilada naturalmente por 20 dias (Fernandes et al., 1987). Em seguida, prepararamse os vasos com a mistura de 3,5 kg de sol o fumigado, ou não fumigado, e $200 \mathrm{~cm}^{3}$ de vermiculita (32 g).

Procedeu-se à calagem ( $\mathrm{V}=92 \%)$ e à adubação para cada vaso, individualmente. Assim, cada vaso recebeu $10 \mathrm{~g}$ de calcário dolomítico (PRNT $95 \%$ ), $180 \mathrm{mg} \mathrm{kg}^{-1}$ de $\mathrm{K}$ no solo (1,3 g por vaso de $\mathrm{KCl}$ ) e fósforo. As doses de fósforo adicionadas foram de: 60, 120, 240 e $480 \mathrm{mg} \mathrm{kg}^{-1}$ de P no solo, na forma de superfosfato simples (180 g kg-1 de $\mathrm{P}_{2} \mathrm{O}_{5}$ ), o que equivaleu à adição de 0,77, 1,54, 3,1 e 6,2 $\mathrm{g} \mathrm{kg}^{-1}$ de superfosfato simples no solo, respectivamente. Prepararam-se, também, vasos sem adição de fósforo.

Após homogeneização, os vasos foram umedecidos até $70 \%$ da capacidade efetiva de retenção de água, permanecendo em incubação por 80 dias. Durante este período, os vasos que receberam terra fumigada foram reinoculados com um filtrado da amostra de solo não fumigada, visando repor a comunidade microbiana original, exceto propágulos de fungos micorrízicos nativos, segundo método utilizado por Azcón et al. (1989).

Os tratamentos foram dispostos em del ineamento em blocos ao acaso, com quatro repetições, segundo um esquema fatorial $2 \times 2 \times 5$ (terra fumigada enão fumigada; com e sem FMA; com quatro doses de adubo fosfático e um nível sem esse adubo). Como adu bação de cobertura, foram aplicados 20 mg de N por vaso, na forma de sulfato de amônio, aos 12, 22,
37, e 47 dias do cultivo em vasos. A umidade do substratofoi mantida em torno de $70 \%$ da capacidade efetiva de retenção de água com adição de água destilada, controlada por meio de pesagens a cada dois ou três dias. A utilização de vermiculita teve por objetivo favorecer a retenção de água.

Nesta fase de vasos, determinaram-se a altura e o número de fol has das plantas aos 86, 101, 116 e 131 dias de idade das plantas. O diâmetro do caule das plantas, por sua vez, foi determinado somente aos 126 dias de idade das plantas. Para cada variável, determinou-se o efeito da inoculação (EI) com o FMA, segundo a equação: $\mathrm{EI}=[$ (variável em plantas inoculadas com FMA - variável em plantas não inoculadas com FMA)/variável em plantas não inoculadas com FMA] x 100.

Os dados foram submetidos à analise de variância para experimentos em blocos casualizados e, para comparação das médias, o teste de Tukey a 1 ou $5 \%$. Para as análises de regressão, utilizou-seo "software Table Curve 2D" (J andel Scientific), sendo escolhida a equação de melhor ajuste de acordo com o maior coeficiente de determinação.

\section{RESULTADOS E DISCUSSÃO}

A inoculação com o FMA e a adição de doses crescentes de fósforo, isoladamente, exerceram efeitos positivos significativos sobrea altura, número de folhas e diâmetro do caule de plantas de mamoeiro. A fumigação do substrato, por sua vez, não exerceu efeitos significativos sobre estas variáveis, muito embora a colonização e a esporulação devidas a fungos micorrízicos nativos tenham sido, em média, de $48 \%$ e de 10,3 esporos (50 $\mathrm{g}$ de substrato)-1 (Auler, 1995). Desta forma, os resultados aqui apresentados referem-se às médias dos dados entre substrato fumigado e não fumigado.

O efeito positivo da inoculação com o F MA sobre a altura foi verificado 35 dias após a inoculação(DMS $=0,41$ ), enquanto para o número de fol has, o efeito foi mais precoce, ou seja, aos 28 dias da inoculação (DMS = 0,43); ambos os efeitos foram de $10 \%$ e ocorreram ainda em fase de bandejas (Figura 1).

$\mathrm{Na}$ fase de vasos, ao final do experimento, os efeitos positivos da micorrização sobre as variáveis do crescimento do mamoei ro ocorreram em todas as doses de fósforo adicionado ao substrato. Estes efeitos positivos foram de 103, 38 e $107 \%$, para a altura, número de fol has e diâmetro do caule, respectivamente, no tratamento sem adição de fósforo (Figuras 2, 3 e 4). A adição de fósforo, por sua vez, aumentou esses efeitos até à dose de $60 \mathrm{mg} \mathrm{kg}^{-1}$ deP no sol o após o que, doses maiores de fósforo reduziram os efeitos da micorrização sobre essas variáveis do crescimento vegetal. 


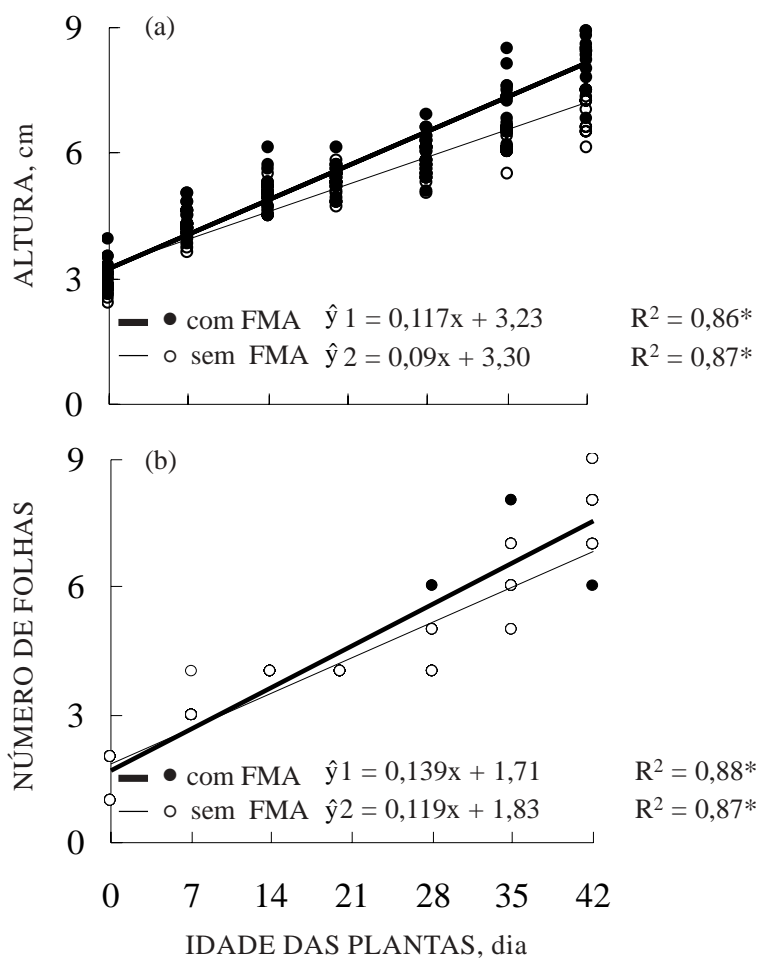

Figura 1. Altura média (a) e número médio de folhas de plantas de mamoeiro (b), inoculadas ou não com Glomus macrocarpum, considerando a idade das plantas, fase de bandeja. Média de dezesseis repetições. (* significativo a $5 \%$ ).

No presente experimento, os maiores efeitos da mi corrizaçào ocorreram no tratamento com a adição de $60 \mathrm{mg} \mathrm{kg}^{-1}$ deP no solo, sendo de 333, 60 e $254 \%$, para a altura, número de fol has e diâmetro do caule, respectivamente. Weber \& Amorim (1994), na associação mamoeiro Sol o-Glomus etunicatum ou Entrophospora colombiana sob vários níveis de fósforo, verificaram que o efeito maior da mi corrização sobrea altura das plantas foi deapenas 148 \% e ocorreu notratamento sem adição de fósforo. Para Silva \& Siqueira (1991), por sua vez, na associação mamoei ro Solo-G. macrocarpum, o efeito maior da inoculação sobre uma variável do crescimento vegetal (peso da matéria seca da parte aérea) foi de 644 \% e ocorreu com a adição de apenas $2 \mathrm{mg} \mathrm{kg}^{-1}$ de $\mathrm{P}$ no substrato.

Esta diversidade de efeitos da mi corrização sobre variáveis do crescimento de plantas tem fundamento nos fatores que regulam as associações micorrízicas. Embora não haja especificidade entre macro e mi crossimbionte, o comportamento das associações micorrízicas, bem como os efeitos e a intensidade destes, variam com a combinação FMA x planta e com os fatores bióticos e abióticos do sistema (Bagyaraj, 1994).

A interação entre a altura e a idade das plantas de mamoeiro (Figura 2), na presença do FMA, obedeceu à função pol inomial quadrática, exceto no tratamento sem adição de fósforo, no qual o comportamento seguiu a função linear. Para as plantas não inoculadas, por sua vez, a função quadrática model ou o comportamento dos dados somentea partir da dose de $240 \mathrm{mg} \mathrm{kg}^{-1}$ deP no solo; na ausência e para as doses mais baixas de fósforo adicionado, o comportamento foi expresso segundo uma função linear.

Para o número de fol has, a interação com a idade das plantas obedeceu à função linear, independentemente da inoculação com o F MA (Figura 3). Nestas funções, os coeficientes angulares foram, geral mente, maiores para as plantas inoculadas, o que corrobora os ganhos em altura eem número de fol has maiores eantecipados em relação às plantas não inoculadas.

$$
\begin{aligned}
& \text { - com FMA } \hat{y}_{1}=0,190 x-2,973 \\
& \mathrm{R}^{2}=0,89^{* *} \\
& \text { ○ sem FMA } \hat{y} 2=0,030 x+7,105 \\
& \mathrm{R}^{2}=0,42 * * \\
& 40 \text { ᄃ } \mathrm{P}=0 \mathrm{mg} \mathrm{kg}^{-1} \\
& 20[0[0
\end{aligned}
$$$$
\text { - } \operatorname{com} \text { FMA } \hat{y} 1=0,013 x^{2}-1,891 x+83,727
$$$$
\mathrm{R}^{2}=0,96 * *
$$$$
\text { ○ sem FMA } \hat{y} 2=0,060 x+4,320
$$$$
\mathrm{R}^{2}=0,52^{* *}
$$

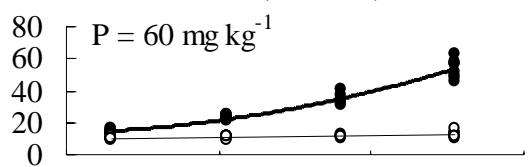

$$
\begin{array}{lll}
\text { - com FMA } & \hat{y} 1=0,012 x^{2}-1,797 x+79,630 & R^{2}=0,93 * * \\
\text { sem FMA } & \hat{y} 2=0,110 x+0,756 & R^{2}=0,77 * *
\end{array}
$$
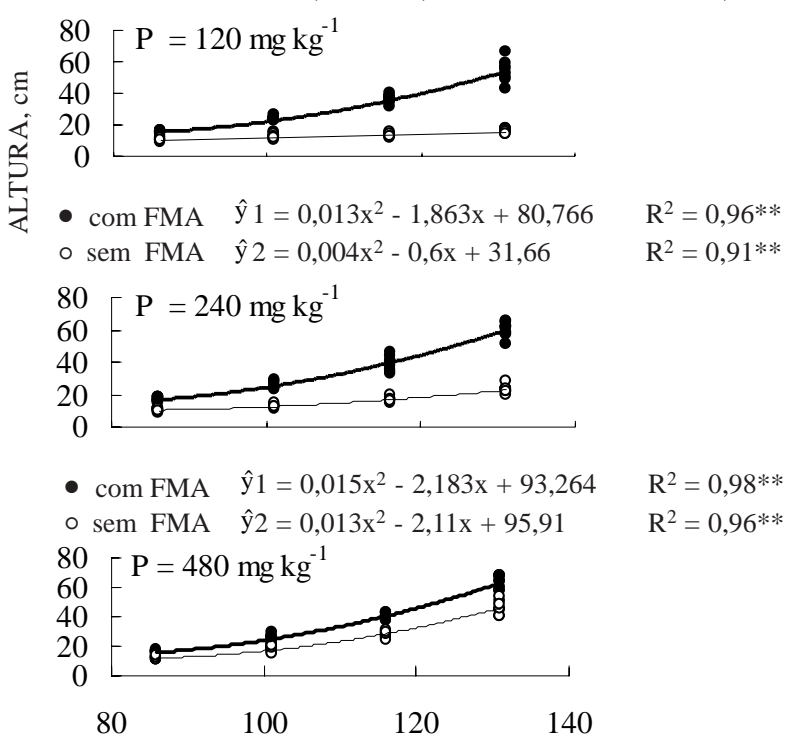

IDADE DAS PLANTAS, dia

Figura 2. Altura média de plantas de mamoeiro, para cada dose de fósforo adicionada ao substrato, com e sem inoculação com Glomus macrocarpum, considerando a idade das plantas, fase de vaso. Média de quatro repetições. (** significativo a $1 \%$ ). 
O efeito do fósforo, nutriente limitante no experimento, sobre o crescimento do mamoeiro seguiu um comportamento esperado (Figura 4). Para as plantas inoculadas, os aumentos médios nas variáveis de crescimento do mamoeiro, conforme a adição de fósforo, foram maiores em relação às plantas não inoculadas atéà dose de $240 \mathrm{mg} \mathrm{kg}^{-1}$ de P no solo. Contudo, a adição de uma dose maior de fósforo ao substrato, $480 \mathrm{mg} \mathrm{kg}^{-1}$ deP no solo, causou uma inversão nos ganhos em crescimento, ou seja, estes foram mai ores para as plantas não inoculadas.

Este comportamento confirma o efeito maior da mi corrização em condições de dose de fósforo abaixo da ótima para o crescimento vegetal (Minhoni et al., 1993; Bagyaraj, 1994; Cardoso Filho et al., 1999; Nogueira \& Cardoso, 2000; Melloni et al., 2000; Moreira \& Siqueira, 2002), ou seja, a dependência

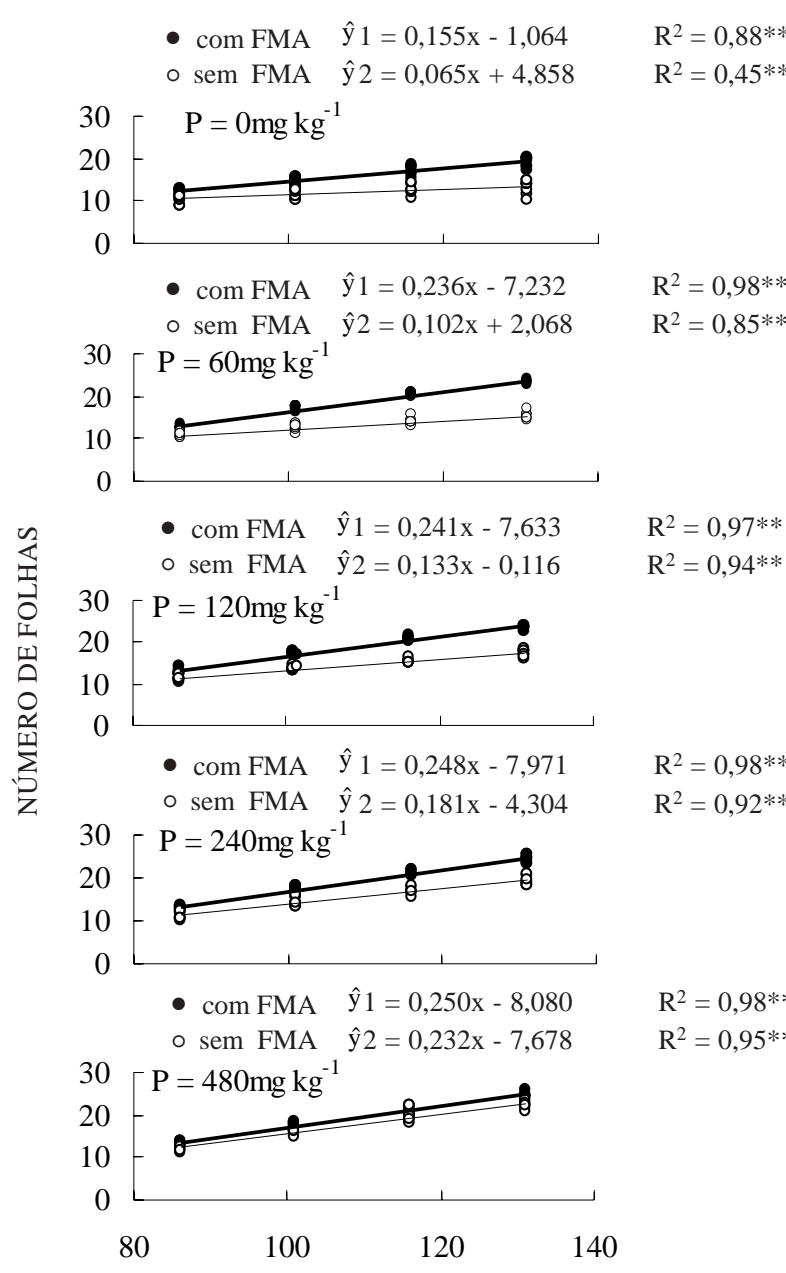

IDADE DAS PLANTAS, dia

Figura 3. Número médio de folhas de plantas de mamoeiro, para cada dose de fósforo adicionada ao substrato, com e sem inoculação com Glomus macrocarpum, considerando a idade das plantas, fase de vaso. Média de quatro repetições.(** significativo a $1 \%)$.

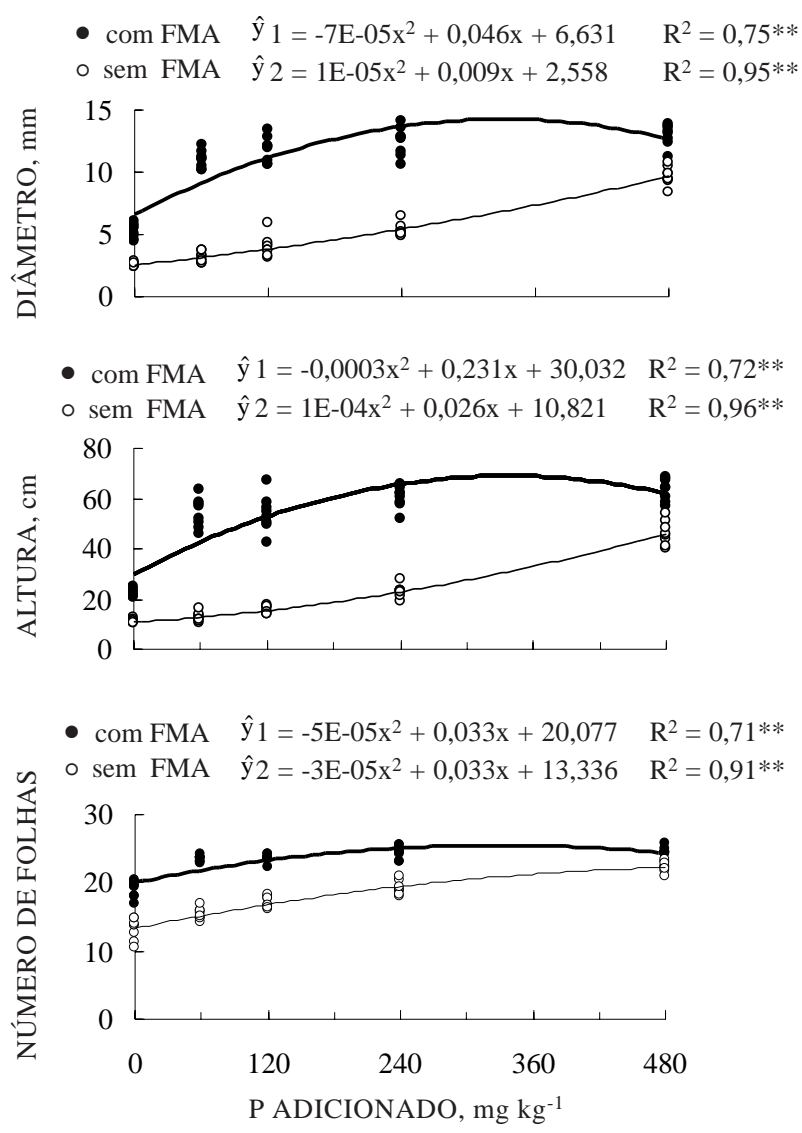

Figura 4. Diâmetro médio (aos 126 dias), altura média (aos 131 dias) e número médio de folhas (aos 131 dias de idade) de plantas de mamoeiro, com e sem inoculação com Glomus macrocarpum, considerando o fósforo adicionado ao substrato, fase de vaso. Média de oito repetições. (**significativo a $1 \%$ ).

micorrízica é inversamente proporcional à disponibilidade de fósforo. O crescimento constante das plantas não inoculadas, considerando o aumento da dose de fósforo adicionado, evidencia a dependência elevada do mamoeiro para este nutriente.

O crescimento das plantas inoculadas em termos de altura, número de fol has e diâmetro de caule, na ausência de adubação fosfática, foi equivalente ao de plantas não inoculadas submetidas à dose de $240 \mathrm{mg} \mathrm{kg}^{-1}$ deP nosolo(Figuras 2, 3 e4). Mohandas (1992) obteve resultados semelhantes, ou seja, a altura de mudas de mamoeiro micorrizadas, na ausência de adubação fosfática, mostrou-se equivalente à de mudas não micorrizadas na dose de $18,4 \mathrm{~g}$ de superfosfato triplo por vaso $(5 \mathrm{~kg})$.

Trindadeet al . (2000) também verificaram efeitos positivos da micorrização, com fungos nativos e exóticos, no crescimento, bem como na eficiência de utilização de fósforo de mudas de mamoeiro. $\mathrm{Na}$ presença de FMAs exóticos (Glomus clarum, 
Gigaspora margarita e Acaul ospora scrobiculata) e nativos (Glomus sp, Gigaspora sp eAcaul ospora sp), o benefício micorrízico sobre o peso de matéria seca da parte aérea situou-se em $63 \%$, enquanto a eficiência média de utilização de fósforo das mudas inoculadas foi o dobro em relação à das mudas não inoculadas.

Efeitos positivos da inoculação com FMA sobreo crescimento e utilização de fósforo são comuns na literatura. Micorrizas revelam uma maior superfície de absorção de água e nutrientes, resultante da presença do micélio externo à raiz, o qual participa e intensifica os efeitos do processo micorrízico (Bagyaraj, 1994; Cardoso Filho et al, 1999; Nogueira \& Cardoso, 2000; Moreira \& Siqueira, 2002). As hifas do mi célio, além do maior contato com as partículas do substrato e do crescimento radial muitas vezes para além da zona de depl eção de nutrientes, podem apresentar atividade el evada na absor ção de fósforo (Silveira \& Cardoso, 1990). Tais características, dentre outras, justificam os efeitos benéficos da micorrização, bem como a maior eficiência na utilização de fósforo em plantas micorrizadas.

A figura 5 apresenta as interações entre o fósforo adicionado e o efeito da inoculação com Glomus macrocarpum, relativas ao diâmetro do caule, à al tura e número de fol has de plantas de mamoeiro. As curvas obtidas, do tipo sigmóide assimétrica, traduzem a dinâmica do efeito da inoculação. O efeito da inoculação, menor na ausência de fósforo adicionado ao substrato, tende a aumentar com o aumento do fósforo adicionado ao substrato, após o que, doses maiores de fósforo inibem o processo micorrízico, diminuindo os benefícios da inoculação.

Esta inibição que o fósforo exerce na mi corrização tem sido explicada por meio de três hipóteses, segundo M oreira \& Siqueira(2002): (a) em condições de suprimento elevado de fósforo, a atividade de fosfatases nas raízes é baixa e, como conseqüência, lecitinas presentes nas raízes ficam livres e ligamse a carboidratos do fungo, inibindo o crescimento deste; (b) em condições de suprimento el evado de fósforo, a biossíntese de fosfolipídeos é aumentada e, como conseqüência, a permeabilidade celular, a exsudação radicular (açúcares e aminoáci dos), a infecção e a colonização radicular são diminuídas; (c) em condições de suprimento el evado de fósforo, a fotossíntese e o dreno de sacarose e, ou, seus derivados para osistema radicular sãomai ores. Esta concentração de sacarose e, ou, de seus derivados el evada no sistema radicular ini biria propágulos do fungo micorrízico, tal como já verificado "in vitro".

Para o presente trabal ho e de acordo com as equações obti das, estima-se que os picos para o efeito da inoculação estariam nas doses de 69, 66 e $36 \mathrm{mg} \mathrm{kg}^{-1}$ de $\mathrm{P}$ no solo para diâmetro de caule, altura e número de fol has, respectivamente.

Os resultados deste trabal ho confirmam o potencial de utilização de fungos micorrízicos

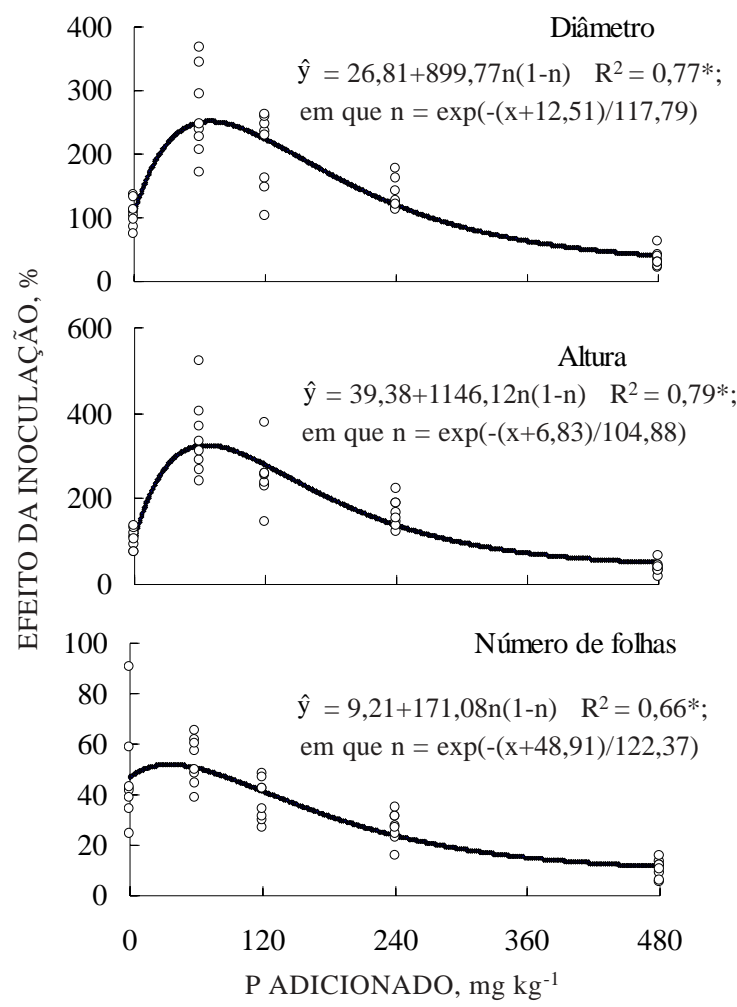

Figura 5. Efeito da inoculação com Glomus macrocarpum para diâmetro médio, $\mathrm{mm}$, (aos 126 dias), altura média, cm, (aos 131 dias) e número médio de folhas (aos 131 dias de idade) de plantas de mamoeiro, considerando o fósforo adicionado ao substrato, fase de vaso. Média de oito repetições. (* significativo a 5 \%).

arbusculares para o mamoeiro, cuja produção de mudas depende, geralmente, da utilização de substrato submetido a tratamentos fitossanitários, além da economia de adubos fosfáticos. Possi bilitam, também, verificar que o mamoeiro necessita de teores elevados de fósforo disponível para o seu crescimentoinicial equea inoculação com $F M A$ pode reduzir substancialmente esta necessidade.

\section{CONCLUSÕES}

1. A inoculação com o FMA G. macrocarpum exerceu efeitos positivos no diâmetro de caule, na altura e no número de folhas de plantas de mamoeiro.

2. Os efeitos positivos da inoculação variaram com a dose de fósforo adicionado ao solo, obedecendo a uma curva tipo sigmóide assimétrica, com picos estimados nas doses de 69, 66 e $36 \mathrm{mg} \mathrm{kg}^{-1}$ de P no solo para diâmetro de caule, altura e número de folhas, respectivamente. 
3. A inoculação reduziu a necessidade de fósforo para plantas de mamoeiro, medida pel os ganhos em diâmetro de caule, altura e número de fol has.

4. A fumigação do solo não interferiu nas medidas dealtura, de número de fol has e dediâmetrodecaule de plantas de mamoeiro com ou sem o FMA G. macrocarpum.

\section{LITE RATURA CITADA}

AZCÓN, R.; RUBIO, R.; MORAES, C. \& TOBAR, R. Interactions between rhizosphere free-living microorganisms and VAM fungi. Agric. Ecosys. Environ., 29:11-15, 1989.

AULER, P.A.M. Desenvolvimento inicial do mamoeiro (Carica papaya L.) relacionado à disponibilidade de fósforo no solo e à colonização pelo fungo micorrízico vesículo-arbuscular Glomus macrocarpum. Botucatu, Universidade Estadual Paulista "J úlio de Mesquita Filho", 1995. 94p. (Tese de Mestrado)

BAGYARAJ , D.J . Vesicular-arbuscular mycorrhiza: application in agriculture. In: NORRIS, J .R.; READ, D.J . \& VARMA, A.K., eds. Techniques for mycorrhizal research: Methods in microbiology. San Diego, Academic Press, 1994. p.819833.

CARDOSO, E.J.B.N.; ANTUNES, V.; SILVEIRA, A.P.D. \& OLIVEIRA, M.H.A. Eficiência de fungos micorrízicos vesículo-arbusculares em porta-enxertos de citros. R. Bras. Ci. Solo, 10:25-30, 1986.

CARDOSO FILHO, J.A.; PACOVSKY, R.S. \& CARDOSO, E.J.B.N. Growth and metabolic activity of the extramatricial mycelium of endomycorrhizal maize plants. R. Bras. Ci. Solo, 23:807-814, 1999.

EMPRESA BRASILEIRA DE PESQUISA AGROPECUÁRIA EMBRAPA. CentroNacional de Pesquisa de Solos. Sistema brasileiro de classificação de solos. Brasília, 1999. 412p.

FERNANDES, A.B.; SIQUEIRA, J .O.; MENEZES, M.A. \& GUEDES, G.A.A. Efeito diferenciado do fósforo sobre o estabel ecimento e efetividade da simbiose endomicorrízica em milho e soja. R. Bras. Ci. Solo, 11:101-108, 1987.

KLEINSCHMIDT, G.D. \& GERDEMANN, J.W. Stunding of citrus seedillings in fumigated nursery soils related to the abscence of endomycorrhizae. Phytopathology, 62:14471453,1972

LIN, M.T. Uso de micorrizas em fruticultura. R. Bras. Frutic., 8:47-55, 1986.

LOPES, E.S.; SIQUEIRA, J .O. \& ZAMBOLIN, L. Caracterização das micorrizas vesicular-arbusculares (MVA) e seus efeitos no crescimento das plantas. R. Bras. Ci. Solo, 7:1-19, 1983.

MARONEK, D.M.; HENDRIX, J.W. \& KIERMAN, J. Mycorrhizal fungi and their importance in horticultural crop production. Hortic. Rev., 3:172-213, 1981.
MELLONI, R.; NOGUEIRA, M.A.; FREIRE, V.F. \& CARDOSO, E.J.B.N. Fósforo adicionado e fungos micorrízicos arbusculares no crescimento e nutrição mineral delimoeirocravo [Citrus limonia (L.) OSBECK]. R. Bras. Ci. Solo, 24:767-775, 2000.

MENGE, J .A.; LARRE, J .; LABANANSKAS, C.K. \& J OHNSON E.L.V. The effect of two mycorrhizal fungi upon growth and nutrition of avocado seedlings grown with six fertilizer treatments. J . Am. Soc. Hortic. Sci., 105:400-404, 1980.

MINHONI, M.T.A.; CARDOSO, E.J .B.N. \& EIRA, A.F. Efeitos da interação de fosfato de rocha, matéria orgânica e fungo micorrízico no crescimento ena absorção de nutrientes pela soja. R. Bras. Ci. Solo, 17:165-171, 1993.

MOHANDAS, S. Effect of VAM inoculation on plant growth, nutrient level and root phosphatase activity in papaya (Carica papaya) cv. Coorg. Honey Dew. Fertil. Res., 31:263267, 1992.

MOREIRA, F.M.S. \& SIQUEIRA, J.O. Microbiologia e bioquímica do solo. Lavras, Universidade Federal de Lavras, 2002. 625p.

NOGUEIRA, M.A. \& CARDOSO, E.J.B.N. Produção de micélio externo por fungos micorrízicos arbusculares ecrescimento da soja em função de doses de fósforo. R. Bras. Ci. Solo, 24:329-338, 2000.

RAIJ, B. van \& QUAGGIO, J.A. Métodos de análise de solo para fins de fertilidade. B. Tec. Inst. Agron., 81:1-31, 1983.

RAMIREZ, B.N.; MITCHEL, D.J. \& SCHENCK. N.C. Establishment and growth effects of three vesicular mycorrhizal fungi on papaya. Mycologia, 67:1039-1041, 1975.

SILVA, L.F.C. \& SIQUEIRA, J.O. Crescimento e teores de nutrientes de mudas de abacateiro, manguei ra e mamoei ro sob influência de diferentes espécies de fungos micorrízicos vesículo-arbusculares. R. Bras. Ci. Solo, 15:283-288, 1991.

SILVEIRA, A.P.D. \& CARDOSO, E.J.B.N. Kinetics of phosphorus uptake, growth and mineral nutrition of mycorrhizal and non-mycorrhizal bean (Phaseol us vul garis L.). In: NORTH AMERICAN CONFERENCE ON MYCORRHIZAE, 8., Wyoming, 1990. Abstracts. Wyoming, 1990. p.269.

SOARES, A.C.F. \& MARTINS, M.A. Influência de fungos micorrízicos arbusculares, associada à adição de compostos fenólicos, no crescimento de mudas de maracujazeiro amarelo (Passiflora edulis f. flavicarpus). R. Bras. Ci. Solo, 24:731-740, 2000.

TRINDADE, A.V.; SIQUEIRA, J .O. \& ALMEIDA, F.P. Eficiência simbiótica de fungos micorrízicos arbusculares em solo não fumigado para mamoeiro. R. Bras. Ci. Solo, 24:505-513, 2000.

WEBER, O.B. \& AMORIM, S.M.C. Adubação fosfática e inoculação de fungos micorrízicos vesículo-arbusculares em mamoeiro "solo". R. Bras. Ci. Solo, 18:187-191, 1994. 
